\section{Resting-State fMRI findings in patients with first-Episode idiopathic epilepsy before and after treatment}

\author{
Peng-Fei Qiao, MD, Guang-Ming Niu, MD.
}

\section{ABSTRACT}

Objectives: To detect resting-state functional MRI (rsfMRI) changes and their relationships with the clinical curative effect of anti-epileptic drugs (AEDs) for complex partial seizures (CPS) in epilepsy patients using the fractional amplitude of low frequency fluctuation (fALFF).

Methods: rfMRI data from 14 CPS patients enrolled between June 2015 and June 2016 in Department of Neurology, Affiliated Hospital of Inner Mongolia Medical University were retrospectively investigated and compared with findings from 14 healthy age-, gender-, handedness-, and education-matched subjects. The patients were treated with first-line antiepileptic drugs for 12 weeks before undergoing a second rfMRI scan. fALFF data were processed using REST and SPM8 software. Whole-brain data were compared between the 2 groups.

Results: The abnormal findings in CPS patients significantly decreased or disappeared after AED treatment.

Conclusion: Measuring fALFF is useful for identifying brain functional changes in patients with first-episode epilepsy before and after treatment. Performing rfMRI is useful for evaluating treatment responses and may provide new insight into the pathophysiological mechanisms of epilepsy.

\section{Neurosciences 2017; Vol. 22 (4): 316-319} doi: 10.17712/nsj.2017.4.20160650

Tiopathic epilepsy is a chronic neurological disease 1 characterized by no organic or metabolic brain abnormalities, varying degrees of autonomic symptoms, consciousness disturbance, and psychiatric symptoms. It may be related to genetic factors, but the pathogenesis is not fully understood. ${ }^{1}$ Patients with this condition experience sudden, transient and recurrent dysfunction of central nervous system caused by super-synchronous abnormal discharges of brain neurons. ${ }^{2}$ Idiopathic epilepsy has complex physiological mechanisms and diverse clinical manifestations. Medication is the primary and most commonly used treatment. Assessing drug efficacy and the development of neurological lesions by non-invasive magnetic resonance imaging (MRI) provides objective evidence for new drug development and clinical treatment. ${ }^{3}$ In this study, fractional amplitude of low frequency fluctuations (fALFF) was measured using resting-state functional MRI (rfMRI) to investigate changes before and after treatment and their relationship with the clinical efficacy of antiepileptic drugs (AEDs).

Methods. This was a retrospective study of 14 patients diagnosed with primary complex partial seizures (CPS) between June 2015 and June 2016 in the Department of Neurology, Affiliated Hospital of Inner Mongolia Medical University. The cohort included 8 males (9-35 years, mean 22.4 years) and 6 females ( $8-40$ years, mean 25.2 years). Fourteen healthy volunteers matched with the patient group in age, gender, handedness, and years of education were selected as controls. This study was approved by the Medical Ethics Committee of Inner Mongolia Medical University Affiliated Hospital. All subjects signed an informed consent form.

Typical primary CPS was diagnosed according to the 2007 diagnostic criteria of the International League Against Epilepsy (ILAE). Only first-episode patients were enrolled to avoid the influences of disease course and drugs. All patients were asked on their detailed medical history by a clinically experienced deputy chief physician or another more highly placed neurological professional. Seizure type was determined based on medical history and electroencephalography (EEG) changes. Neurological examinations were performed to exclude serious physical diseases and complications, and conventional brain MRI scans were taken to exclude apparent structural abnormalities.

The rfMRI was performed in both the patient and control groups using a GE Discovery 750 3.0T superconductive MRI scanner (GE Healthcare, Chicago, IL, USA). A second scan was performed in the patient group after 12 weeks of treatment with the conventional first-line AED carbamazepine.

Subjects laid awake on the table with noise-blocking headphones and were instructed to close their eyes, keep their heads still, and try not to think or perform specific cognitive tasks. Scanning did not begin until subjects adapted to the environment.

Disclosure. Authors have no conflict of interests, and the work was not supported or funded by any drug company. 
Table 1 - Regions showing increased fALFF between healthy subjects and untreated epilepsy patients.

\begin{tabular}{lcccc}
\hline Regions & $\mathrm{L} / \mathrm{R}$ & $\mathrm{MNI}(\mathrm{X}, \mathrm{Y}, \mathrm{Z})$ & Voxels $\left(\mathrm{mm}^{3}\right)$ & $\mathrm{t}-$ Score \\
\hline Thalamus & $\mathrm{R}$ & $14,-30,10$ & 28 & 2.9962 \\
Thalamus & $\mathrm{L}$ & $-12,-28,9$ & 35 & 3.6621 \\
Posterior cingulate & $\mathrm{R}$ & $-13,-50,16$ & 31 & 3.5521 \\
Posterior cingulate & $\mathrm{L}$ & $12,-45,18$ & 28 & 2.7536 \\
Angular gyrus & $\mathrm{L}$ & $-56,-47,39$ & 35 & 3.8926 \\
Inferior parietal lobule & $\mathrm{L}$ & $-50,-27,25$ & 28 & 3.6249 \\
\hline \multicolumn{2}{c}{ R - right, L - left, MIN - Montreal Neurological Institute coordinate, } \\
\multicolumn{4}{c}{ fALFF -fractional amplitude of low frequency fluctuation } \\
\hline
\end{tabular}

Table 2 - Regions showing decreased fALFF between healthy subjects and untreated epilepsy patients.

\begin{tabular}{lcccc}
\hline Regions & L/R & MNI (X,Y,Z) & Voxels $\left(\mathrm{mm}^{3}\right)$ & t-Score \\
\hline Medial prefrontal lobe & $\mathrm{R}$ & $3,55,25$ & 26 & -2.7786 \\
Medial prefrontal lobe & $\mathrm{L}$ & $-7,63,15$ & 38 & -3.6603 \\
Anterior cingulate & $\mathrm{R}$ & $3,9,24$ & 19 & -2.9482 \\
Anterior cingulate & $\mathrm{L}$ & $-7,15,26$ & 21 & -3.1325 \\
\hline R - right, L - left, MIN - Montreal Neurological Institute coordinate, \\
$\quad$ fALFF -fractional amplitude of low frequency fluctuation \\
\hline
\end{tabular}

Table 3 - Regions showing increased fALFF between healthy subjects and treated epilepsy patients .

\begin{tabular}{lcccc}
\hline Regions & L/R & MNI $(\mathrm{X}, \mathrm{Y}, \mathrm{Z})$ & Voxels $\left(\mathrm{mm}^{3}\right)$ & $\mathrm{t}$-Score \\
\hline Angular gyrus & $\mathrm{L}$ & $-54,-45,37$ & 13 & 2.1689 \\
Inferior parietal lobule & $\mathrm{L}$ & $-55,-23,27$ & 38 & 3.6649 \\
Frontal lobe & $\mathrm{L}$ & $-42,30,15$ & 20 & 2.0922 \\
Frontal lobe & $\mathrm{R}$ & $40,33,19$ & 23 & 2.6839 \\
Precuneus & $\mathrm{L}$ & $-7,-43,46$ & 26 & 3.5513 \\
Precuneus & $\mathrm{R}$ & $9,-52,35$ & 20 & 3.0015 \\
Cuneus & $\mathrm{L}$ & $-8,-61,53$ & 35 & 2.5427 \\
\hline \multicolumn{5}{c}{$\mathrm{R}$ - right, L- left, } \\
\hline
\end{tabular}

Functional scanning was performed using echo planar imaging with gradient recalled echo sequences (field of view $24 \mathrm{~cm} \times 24 \mathrm{~cm}, 64 \times 64$ matrix, flip angle $90^{\circ}$, repetition time $2000 \mathrm{~ms}$, echo time $30 \mathrm{~ms}$ ) with whole-brain coverage using 28 oblique axial 4-mm slices with $4-\mathrm{mm}$ spaces. The first 10 images were excluded owing to T1 equilibrium effects. Resting-state scanning lasted for $512 \mathrm{~s}$, producing 256 brain volume data sets. rfMRI data of the 256 volumes of each subject were used for analysis. Data preprocessing was performed using the SPM8 software package (statistical parametric mapping, http://www.fil.ion.ucl.ac.uk/ $\mathrm{spm} /$ ). After slice timing, realignment, normalization, smoothing, and filtering, the time series of each voxel was transformed to the frequency domain to obtain the power spectrum. Next, we calculated the square root at each frequency of the power spectrum to obtain the averaged square root across $0.01-0.08 \mathrm{~Hz}$ at each voxel. This averaged square root was taken as the ALFF, and fALFF is calculated as the ratio of ALFF between 0.01 and $0.08 \mathrm{~Hz}$ to the ALFF over the entire frequency range. Lastly, the statistical parametric brain maps of the amplitude were obtained to describe spontaneous activity of voxel intensity. The MATLAB-based restingstate data analysis tool REST (www. restfmri.net) was used to assess the fALFF maps. Voxelwise two-sample t-tests were employed to identify the increased or decreased activity regions of CPS patients compared to healthy controls before and after treatment. The t-value was set to $p<0.05$ after Alphasim correlation.

Results. Compared with the control group, the untreated patient group showed significantly higher fALFF in the bilateral thalami, posterior cingulate, left angular gyrus, and inferior parietal lobule and significantly reduced fALFF in the bilateral medial prefrontal lobes and anterior cingulate. After treatment, abnormally activated areas decreased or disappeared in the thalamus and angular gyrus and increased in the left inferior parietal lobule. In addition, newly activated brain areas were detected in the bilateral frontal lobes, precuneus, and left cuneus (Tables 1, 2, and 3).

Discussion. Low-frequency amplitude analysis was first developed and applied to resting-state fMRI research by Zang et al. $^{4}$ It reflects the amplitude of blood-oxygen-level dependent signal changes from baseline. Data between 0.01 and $0.08 \mathrm{~Hz}$ were included in the analysis. Spontaneous activity of brain regions in the resting state is reflected from the perspective of energy, and neuronal excitability and metabolism are measured by $\mathrm{ALFF}^{5}$ to identify functional changes in the brains of patients with epilepsy. However, ALFF is sensitive to physiological noise. To this end, Zou et al. ${ }^{6}$ proposed the use of fALFF. This approach effectively inhibits interference from the large vascular compartment and ventricular system, improving the specificity of detecting spontaneous brain activity and better reflecting functional brain characteristics.

The results of this study show that compared with the control group, patients with untreated CPS exhibited significantly higher fALFF in the bilateral thalami, posterior cingulate, left angular gyrus, and inferior parietal lobule and significantly reduced fALFF in the bilateral medial prefrontal lobes and anterior cingulate. These findings are consistent with those of previous studies. ${ }^{7}$ These regions were distributed in a roughly symmetrical pattern. Some inconsistencies in affected brain regions may be related to differences in 
age, gender, and research methods. The observation of abnormal brain activity over a wide area once again demonstrates that seizure activity begins and spreads based on abnormalities in an extensive neural network. The thalamus is the brain's most important sensory conduction and relay station and plays a role in the initial facilitation and spread of seizure activity. ${ }^{8,9}$ It is composed of several gray nuclei, each with a different role. It is most strongly implicated in propagating partial seizures. The posterior cingulate is mainly involved in regulating brain activity related to memory, ${ }^{10}$ but it is also an important part of the intracranial spreading network of seizure activity. Metabolic activity in the posterior cingulate increases with seizure activity. This affects its own functioning, which, in turn, explains the confusion and blackouts experienced during seizures and the subsequent development into amnesia and other mental disorders over time. The angular gyrus is the human visual language center, also known as the reading center. ${ }^{11}$ Damage to this area is presumably associated with cognitive language dysfunction in epilepsy. ${ }^{12}$ In this study, these brain regions were significantly activated with increased neuronal excitability before AED treatment. We believe that the regulation and spread of epileptiform discharges are directly related to dysfunction of these brain areas. However, activation in these areas was significantly reduced or disappeared after treatment, suggesting that the abnormalities normalized. Zhang et $\mathrm{al}^{13}$ analyzed EEG characteristics in children with benign epilepsy and their correlations with cognitive level before and after treatment. They reported significantly reduced discharges and significantly improved EEG findings after treatment. The findings of Zhang et $\mathrm{al}^{13}$ and the present study suggest that AED treatment can substantially reduce abnormal brain activity in patients with seizures.

Importantly, activity in some small areas was not significantly affected by treatment. The activated area in the left inferior parietal lobule actually became larger. Damage to the inferior parietal lobule is related to limited memory capacity. Zhang et $\mathrm{al}^{13}$ reported that patients with inferior parietal lobe damage had obvious memory impairment and a significantly lower memory quotient than the control group. There may be 2 reasons for persistent and worsened abnormalities. Firstly, prolonged seizures cause hypoxia and consequent irreversible brain damage. This could be a characteristic of idiopathic epilepsy that serves as the neural basis of seizure recurrence. Secondly, it may be related to the effects of long-term medication. The pharmacological mechanism requires further investigation.
Patients with untreated CPS had significantly reduced AALFF in the bilateral medial prefrontal lobes and anterior cingulate compared with the control group. These abnormalities reduced or disappeared after treatment. Activity in the bilateral medial prefrontal lobes and anterior cingulate was inhibited before treatment, which resolved after 12 weeks of AED use. However, newly activated brain areas included the bilateral frontal lobes, precuneus, and left cuneus. These regions belong to or are near the "default mode network" (DMN). ${ }^{14}$ This suggests that as epileptic activity initiates and spreads, leading to abnormal brain activity, the DMN is inhibited, resulting in negative activation of the network. When the network can maintain high activity after treatment, this manifests as normal activation at rest. These results further support the hypothesis that AEDs decrease or reverse epileptic activity in specific brain regions.

Follow-up assessments confirmed that patients had no or significantly decreased seizures after treatment. This suggests that AEDs partially reversed abnormal brain function while improving the symptoms. Jansen et $\mathrm{al}^{15}$ used fMRI to examine the effects of topiramate on abnormal brain activity in patients with cognitive language impairment due to epilepsy. They found obvious activation of the prefrontal lobe in the treatment group compared with the control group, indicating that the drug normalized brain function, which is consistent with the findings of the present study. It should be noted that the sample size was small in this study. As a result, stratified analysis by age, gender, education level, and other factors was not performed. The results therefore require further verification in more subjects. Although fALFF data can be obtained from the whole brain, functional characteristics of specific brain regions might be neglected with this approach.

In conclusion, there are few comparative rfMRI studies on brain network abnormalities before and after epilepsy treatment. To avoid the influence of medication and disease course, only first-episode patients were enrolled in this study. fALFF data of the whole brain were analyzed and compared. These are innovations of the present study. In this way, the changes in brain function before and after treatment can be better revealed, and the treatment response in epilepsy and efficacy of antiepileptic drugs can be objectively assessed, thus contributing to further research of the complex pathophysiology of epilepsy. Therefore, the sample size needs to be increased in future studies, and multimodal functional imaging should be combined with fALFF to improve result credibility and support clinical and research efforts. 
Received 18th December 2017. Accepted 1st August 2017.

From the Department of Magnetic Resonance Imaging, Affiliated hospital of Inner Mongolia Medical University, Hohhot, Inner Mongolia Autonomous Region, People's Republic of China. Address correspondence and reprints request to: Dr. Guang-ming Niu, Department of Magnetic Resonance Imaging, Affiliated hospital of Inner Mongolia Medical University, Inner Mongolia Autonomous Region, People's Republic of China.E-mail:24853170@qq.com ORCID ID: orcid.org/0000-0001-5915-0837

\section{References}

1. Qiao PF, Gao PY, Dai JP, Niu GM. Research progress on resting state fMRI of epilepsy. Brain Dev 2012; 34: 8-12.

2. Fisher RS, Acevedo C, Arzimanoglou A, Bogacz A, Cross $\mathrm{JH}$, Elger CE, et al. ILAE official report: a practical clinical definition of epilepsy. Epilepsia 2014; 55: 475-482.

3. Qiao P, Niu GM, Han X. Resting state fMRI research of the idiopathic complex partial seizures epilepsy. Chinese J Clinicians 2013; 7: 75-78.

4. Zang YF, He Y, Zhu CZ, Cao QJ, Sui MQ, Liang M, et al. Altered baseline brain activity in children with ADHD revealed by resting-state functional MRI. Brain Dev 2007; 29: 83-91.

5. Guo WB, Liu F, Xue ZM, Xu XJ, Wu RR, Ma CQ, et al. Alterations of the amplitude of low-frequency fluctuations in treatment-resistant and treatment response depression: a resting-state fMRI study. Prog Neuropsychopharmacol Biol Psychiatry 2012; 37: 153-160.

6. Zou QH, Zhu CZ, Yang Y, Zuo XN, Long XY, Cao QJ, et al. An improved approach to detection of amplitude of lowfrequency fluctuation (ALFF) for resting-state fMRI: fractional ALFF. J Neurosci Methods 2008; 172: 137-141.
7. Qiao PF, Gao Y, Xie SH, Niu GM. Progress in Functional Magnetic Resonance Imaging (fMRI) for Study and Treatment of Depression. Med Sci Rev. 2016;3:85-89.

8. Qiao PF, Niu GM. The application of ALFF to the idiopathic complex partial seizures epilepsy of teenagers. Neurol Psychiatry Brain Res 2015; 21: 27-32.

9. Archer JS, Abbott DF, Waites AB, Jackson GD. fMRI "deactivation" of the posterior cingulate during generalized spike and wave. Neuroimage 2003; 20: 1915-1922.

10. Kremer S, Braun M, Kahane P, Guillemin F, Le Bas JF, Benabid AL. MRI morphological and volumetric study of the cingulate gyrus and its relevance in partial epileptic patients. Epileptic Disord 2003; 5: 101-107.

11. Moeller F, Siebner HR, Wolff S, Muhle H, Boor R, Granert $\mathrm{O}$, et al. Changes in activity of striato-thalamo-cortical network precede generalized spike wave discharges. Neuroimage 2008; 39: 1839-1849.

12. Zhang Y, Yan X, Gao Y, Xu D, Wu J, Li Y. A preliminary study of epilepsy in children using diffusional kurtosis imaging. Clin Neuroradiol 2013; 23: 293-300.

13. Zhang Z, Xu Q, Lu G, Liao W, Wang Z, Jiao Q. Dynamic functional MRI study of absence seizures. Chinese JMRI 2013; 4: 3-7.

14. Raichle ME, MacLeod AM, Snyder AZ, Powers WJ, Gusnard DA, Shulman GL. A default mode of brain function. Proc Natl Acad Sci U S A 2001; 98: 676-682.

15. Jansen JF, Aldenkamp AP, Marian Majoie HJ, Reijs RP, de Krom MC, Hofman PA, et al. Functional MRI reveals declined prefrontal cortex activation in patients with epilepsy on topiramate therapy. Epilepsy Behav 2006; 9: 181-185.

\section{Do you have any comments or questions? Agree or disagree with published articles?}

The correspondence section within the journal is a forum to comment on any of the articles published in the journal. Correspondence will not be sent for peer review, and will only be edited for the use of appropriate language. All correspondence should be submitted and published within 6 months from the date of the original publication.

Please submit your correspondence through the journal website (www.neurosciencesjournal.org), and don't forget to clearly state the title of the original publication, and your contact details. 